\title{
CARACTERIZAÇÃO DO CONSUMO DE LEITE EM IDOSOS
}

\section{MILK CONSUMPTION PROFILE IN ELDERLY PEOPLE}

\author{
Adriana Passanha ${ }^{1}$ \\ Heloisa de Souza Garcia ${ }^{1}$ \\ Ana Maria Cervato-Mancuso ${ }^{2}$ \\ Samantha Caesar de Andrade ${ }^{3}$ \\ Viviane Laudelino Vieira ${ }^{3}$
}

Passanha A. et al. Caracterização do consumo de leite em idosos. Rev Bras Cresc e Desenv Hum 2011; 21(2): 319-326.

\section{RESUMO}

O idoso está exposto a modificações em seu equilíbrio nutricional devido a fatores fisiológicos e socioeconômicos, uso de fármacos, doenças, e/ou dificuldades na obtenção de alimentos, bem como sua forma de preparo. Isso pode tornar a ingestão alimentar inadequada, levando ao consumo insatisfatório de diversos alimentos, inclusive o leite. $\mathrm{O}$ objetivo é caracterizar aspectos relacionados à ingestão de leite por idosos. Trata-se de um estudo transversal com idosos voluntários de ambos os sexos, vacinados em uma Unidade Básica de Saúde. Os participantes foram questionados sobre a quantidade consumida diariamente de leite. Realizou-se descrição das variáveis por meio de frequências, desvio padrão e média; utilizou-se o teste qui-quadrado, com significância indicada por $p<0,05$ para verificação de associação entre elas. Foram entrevistados 300 idosos, sendo $67,0 \%$ do grupo d"75 anos e 64,3\% do sexo feminino. Os idosos do grupo $>75$ anos e aqueles do sexo feminino referiram consumo maior de leite. Entretanto, a média de ingestão diária entre os idosos foi de 275,50mL ( $\mathrm{dp}=226,67)$. Ao comparar os valores de ingestão média com o preconizado pelo Guia alimentar para a população brasileira, observa-se que nenhum grupo estudado atingiu tal recomendação, desconsiderando os derivados do leite. As diferenças de ingestão entre os grupos foram estatisticamente significantes. Considerando as vantagens de incluir este alimento na dieta faz-se necessário desenvolver ações voltadas para esse tema, visto que muitos apresentaram atitudes negativas frente a esse assunto.

Palavras-chave: saúde do idoso; nutrição do idoso; leite; ventros de saúde.

\footnotetext{
Nutricionistas, Aprimorandas em Nutrição em Saúde Pública pela Faculdade de Saúde Pública - USP.

Professora Doutora do Departamento de Nutrição da Faculdade de Saúde Pública - USP.

Nutricionistas do CRNUTRI - Centro de Referência para a Prevenção e Controle das Doenças Associadas à Nutrição/ Faculdade de Saúde Pública - USP.

Trabalho realizado no Centro de Saúde Escola Geraldo de Paula Souza, localizado na Faculdade de Saúde Pública - USP. Faculdade de Saúde Pública - USP. Departamento de Nutrição. Avenida Doutor Arnaldo, 715. Cerqueira César, São Paulo SP - Brasil. CEP: 01246-904

Correspondência para: Adriana Passanha - e-mail: adriana.passanha@usp.br

Conflito de interesse: nada a declarar
} 


\begin{abstract}
The elderly are exposed to changes in nutritional balance due to socioeconomic and physiological factors, use of medicines, illness, and/or difficult in obtaining and preparing food. These factors can lead to an inadequate dietary habit and to an inappropriate consumption of several foods, including milk. The aim of this study is to characterize aspects of milk consumption of the elderly vaccinated in the $11^{\text {th }}$ National Campaign of Vaccination of the Elderly. This is a cross-sectional study in elderly volunteers of both sexes vaccinated in a School Health Center. Participants were asked about daily milk intake. Variables description was performed by frequency, standard deviation and mean; the chisquare test was used, with significance indicated by $\mathrm{p}<0.05$ to verify the association between them. Three hundred elderly were interviewed, which whom $33.0 \%$ were more than 75 years old ( $>75$ years old group) and $64.3 \%$ were female. The $>75$ years old group and women reported greater consumption of milk. However, the average daily intake was 275.5 $\mathrm{mL}(\mathrm{SD}=226.67)$. By comparing the values with the average intake recommended by the Brazilian Food Guide, it's possible to observe that no group reached the recommendation, excluding the dairy products. Differences between the groups intake were statistically significant. Considering the advantages of including this food in the diet it is necessary to develop actions related to this issue, since many of the elderly showed negative attitudes.
\end{abstract}

Key words: health of the elderly; elderly nutrition; milk; health centers.

\section{INTRODUÇÃO}

A população brasileira vem envelhecendo de forma rápida e intensa; estima-se que em 2020 o número de idosos alcance 25 milhões ${ }^{1}$. O envelhecimento populacional, característica da transição demográfica, acarreta profundas implicações sobre as políticas sociais e representa um dos maiores desafios da saúde pública contemporânea ${ }^{2}$, fazendo do envelhecimento um fenômeno que precisa de ampla discussão ${ }^{3}$.

Por razões socioeconômicas, fisiológicas, uso de fármacos, diminuição da percepção sensorial, alterações mentais, além de outras doenças que possam reduzir o apetite e diminuir ou aumentar a necessidade de nutrientes $^{4}$, o idoso está exposto a modificações de seu equilíbrio nutricional ${ }^{5,6,7}$.

Acrescenta-se ainda a estas razões a dificuldade na obtenção e na forma de preparo dos alimentos tornando a inadequação da ingestão alimentar mais evidente. Um dos alimentos diretamente afetado por estes fatores é o leite ${ }^{6}$.

O leite é um alimento importante para o desenvolvimento humano, sendo boa fonte de proteínas, vitaminas (em especial A e D), magnésio, cálcio, potássio e água ${ }^{8,9,10,11}$. O Guia Alimentar para a População Brasileira ${ }^{12}$ preconiza a ingestão de 3 porções de leite e derivados por dia; no caso do leite, especificamente, 1 porção corresponde a 1 copo de $200 \mathrm{~mL}$. Esta recomendação é válida também para a população idosa ${ }^{7,13}$.

Dado a vulnerabilidade nutricional verificada entre os idosos, que aponta para a necessidade de cuidados especiais e orientações sobre alimentação adequada ${ }^{14}$, a Campanha Nacional de Vacinação do Idoso consiste em uma maneira para se desenvolver atividades de promoção da alimentação saudável e saúde, que sejam coerentes às características desta população ${ }^{15,16}$. Ainda que o leite seja um alimento com 
importante fonte de nutrientes para os idosos, são poucos os trabalhos científicos que avaliam seu consumo por esta população.

Assim, o objetivo do presente estudo é caracterizar aspectos relacionados à ingestão de leite por idosos.

\section{MÉTODO}

Trata-se de um estudo transversal, com amostragem não probabilística constituída por voluntários idosos, de ambos os sexos, vacinados no Centro de Saúde Escola Geraldo de Paula Souza, localizado na zona oeste do município de São Paulo.

Foram considerados idosos indivíduos com 60 anos de idade ou mais, conforme descrição do Estatuto do Idoso ${ }^{17}$.

O estudo foi realizado em maio de 2009, período correspondente à $11^{\mathrm{a}}$ edição da Campanha Nacional de Vacinação do Idoso. Os idosos foram abordados enquanto aguardavam na fila de cadastro de vacinação. Na ausência de fila, os indivíduos foram entrevistados após serem vacinados.

O diagnóstico referente à ingestão de leite esteve inserido em atividade promotora do consumo de líquidos. Para a coleta de dados, foi utilizado questionário elaborado a partir de outros instrumentos que abordaram o mesmo tema ${ }^{18,19}$.

Foram utilizadas variáveis qualitativas e quantitativas para caracterizar o perfil do idoso atendido na campanha de vacinação pela unidade de saúde, sendo elas: sexo; idade e quantidade diária de ingestão de leite.

A quantidade diária de ingestão de leite foi coletada em medida caseira (copos). Para avaliar esses valores, foi realizada a conversão para mililitros $(\mathrm{mL})$ comparando-se com a medida de referência utilizada pelo Guia Alimentar para a População Brasileira, em que 1 copo de leite equivale a $200 \mathrm{~mL}^{12}$.

As faixas etárias dos participantes foram previamente categorizadas em $<75$ anos e $>75$ anos, como grupos de menor e maior vulnerabilidade para o desenvolvimento de doenças, respectivamente, de acordo com o preconizado pela American College of Cardiology e American Heart Association ${ }^{20}$.

Para caracterização da população de estudo, foi feita descrição das variáveis por meio de frequências, desvio padrão e média. Para verificar a diferença entre as variáveis foi realizada utilizando o teste Qui-quadrado e a comparação por médias, com nível de significância indicado por $\mathrm{p}<0,05$.

Os dados foram tabulados e processados por meio dos softwares Microsoft Excel 2003 e EpiInfo versão 6.04, de 2001.

\section{RESULTADOS}

Foram entrevistados 300 idosos, sendo que $67,0 \%$ tinham idade menor ou igual a 75 anos. A idade dos participantes variou de $60 \mathrm{a}$ 96 anos, com média igual a 71,8 anos $(\mathrm{dp}=7,92)$. A maioria da população pertencia ao sexo feminino $(64,3 \%)$.

A média de ingestão entre os idosos estudados foi de 275,50mL ( $\mathrm{dp}=226,67)$. Para os idosos do sexo masculino, a ingestão média de leite foi de 250,5mL ( $d p=249,29)$; para o sexo feminino foi de $289,4 \mathrm{~mL}(\mathrm{dp}=212,51)$. Comparando o consumo entre as faixas etárias, para aqueles com 75 anos ou menos a média de ingestão foi de $238,3 \mathrm{~mL}(\mathrm{dp}=197,67)$, e para os que possuem mais de 75 anos foi de $351,0 \mathrm{~mL}$ $(\mathrm{dp}=261,56)$. Ambas as análises foram estatisticamente significantes (tabela 1).

\section{DISCUSSÃO}

Conforme observado neste estudo, os idosos com mais de 75 anos e aqueles do sexo feminino referiram consumo maior de leite. Ainda assim, os idosos estudados apresentaram valores baixos de ingestão deste alimento, 
Tabela 1: Média e desvio-padrão da ingestão de leite por idosos segundo sexo e faixa etária. $11^{\mathrm{a}}$ edição da Campanha Nacional de Vacinação do Idoso. Centro de Saúde Escola Geraldo de Paula Souza (São Paulo), 2009

\begin{tabular}{ccccc}
\hline Variáveis & & Ingestão média $(\mathbf{m L})$ & DP & p \\
\hline Sexo & Masculino $(\mathrm{n}=107)$ & 250,5 & 249,29 & $0,03^{*}$ \\
& Feminino $(\mathrm{n}=193)$ & 289,4 & 212,51 & \\
\hline Idade & $<75$ anos $(\mathrm{n}=201)$ & 238,3 & 197,67 & $0,00^{*}$ \\
& $>75$ anos $(\mathrm{n}=99)$ & 351,0 & 261,56 & \\
\hline$* \mathrm{n}<0,05$ & & & &
\end{tabular}

uma vez que a média de ingestão diária entre os idosos foi cerca de um copo de leite.

Outros trabalhos ${ }^{21,23}$ que avaliaram o consumo de leite entre idosos encontraram resultados semelhantes. Elbon et al. ${ }^{23}$ observaram que $49,0 \%$ dos entrevistados consumiam menos de 1 porção de leite $(200 \mathrm{~mL})$ por dia, corroborando com o estudo de Fischer et al..$^{22}$ que encontraram o mesmo resultado para $1 / 3$ dos participantes.

Não foram encontrados estudos que analisassem a ingestão de leite diferenciando-a por sexo. O maior consumo observado entre as mulheres pode ser explicado pela maior preocupação com a sua saúde, realização de exames preventivos e, por isso, apresentam-se mais sensibilizadas sobre os benefícios deste produto. Kurnik et al. ${ }^{24}$, que avaliou o consumo de leite e derivados por idosas fisicamente ativas, também sugerem que as mulheres estão mais preocupadas com a saúde, pois nenhum idoso do sexo masculino se dispôs a participar da pesquisa e conhecer o próprio consumo de leite e derivados. Nesta pesquisa, observou-se que a grande maioria das idosas consumiam ao menos 1 porção diária de leite, consumo semelhante ao apresentado pelas idosas do presente estudo.

Já a ingestão mais elevada entre idosos com maior idade também foi observada por Ryan et al. ${ }^{21} \mathrm{e}$ Fischer et al. ${ }^{22}$. É possível inferir que tais resultados foram encontrados devido ao fato de que os idosos com maior idade utilizam mais os serviços de saúde e, portanto, recebem mais orientações, incluindo as que incentivam o consumo deste alimento. Outra possível explicação é que estes idosos tendem a morar sozinhos e, pela praticidade, podem substituir refeições principais por lanches, aumentando a ingestão de leite.

De acordo com a Pesquisa de Orçamentos Familiares de 2002-2003 ${ }^{25}$, que buscou analisar a evolução das estruturas de consumo alimentar da população brasileira, o consumo de leite apresentou queda de $40 \%$ em relação à Pesquisa realizada em 1987-1988, sugerindo que a ingestão deste alimento está aquém do recomendado. Resultados encontrados por Gerrior et al. ${ }^{26}$ corroboram este achado: entre 1970 e 1997, o consumo de leite pela população americana mostrou redução de aproximadamente $25 \%$.

Uma possível justificativa para a menor aquisição do leite é a ideia de que este alimento não é necessário a partir da vida adulta ${ }^{14}$. Desde a década de 70 há trabalhos que argumentam sobre a falta de necessidade do leite após o desmame e até mesmo sobre aspectos negativos de seu consumo ${ }^{27}$. No entanto, muitos outros trabalhos vêm sendo publicados mostrando que estes aspectos negativos possuem ocorrência mínima e não chegam a causar efeitos deletérios no organismo, além de apresentarem a importância da ingestão diária de leite e os benefícios que este alimento proporciona em todas as fases da vida?

Outro motivo possível para redução da aquisição do leite é o fato de alguns idosos apresentarem intolerância à lactose ${ }^{14}$. Este quadro, muitas vezes, é comum no processo 
de envelhecimento, onde ocorre a diminuição da produção da enzima lactase ${ }^{28}$, fazendo com que os idosos reduzam o consumo de leite ${ }^{14}$. Estudo realizado em 2004 na cidade de Joinville (Santa Catarina - Brasil) que avaliou a prevalência de intolerância à lactose entre indivíduos de todas as faixas etárias mostrou que, analisando apenas os idosos estudados, a prevalência da intolerância foi de $28,75 \%$; considerando a população total do estudo (ou seja, todas as faixas etárias), a prevalência desta intolerância nos idosos foi de $1,93 \%{ }^{29}$. No entanto, deve-se evitar a exclusão total da lactose na dieta, pois pode ocorrer prejuízo nutricional de cálcio, fósforo e vitaminas $^{30}$. Além disto, a maioria das pessoas com esta intolerância pode ingerir $12 \mathrm{~g} / \mathrm{dia}$ de lactose (presente em um copo de leite) sem apresentar sintomas adversos ${ }^{28}$.

No presente estudo, os resultados encontrados mostram-se preocupantes, apesar de existirem diversas vantagens e facilidades para o consumo desta bebida. O leite é considerado um alimento altamente versátil, pois ao mesmo tempo em que é uma bebida pronta para ser consumida, possui outras formas de consumo, tais como matéria-prima para inúmeros produtos da indústria alimentícia e ingrediente culinário responsável por variadas preparações ${ }^{8,31}$.

Com a elevada diversidade deste produto, em diferentes versões de embalagem e marcas e preços variados, é possível encontrar opções de baixo custo desse alimento ${ }^{31,32}$. Considerando este fator, o leite deveria conquistar uma significativa parcela do mercado consumidor $^{31,32}$, já que a disponibilidade deste alimento aumentou para a população em geral $^{31,33}$.

O leite é a fonte mais econômica de certos nutrientes, como cálcio, potássio e magnésio ${ }^{9}$. Nos países com indústrias de laticínios, o leite é um dos alimentos com menor custo por caloria. Assim, o consumo de leite torna-se custo-eficiente, bem como apresenta maior relação custo-benefício ${ }^{34,35}$.
O leite é um dos poucos alimentos que contêm proteínas com todos os aminoácidos essenciais; ou seja, esse alimento oferece proteínas de alto valor biológico, necessárias para atender às demandas fisiológicas também nesta faixa etária ${ }^{7,36}$. Já a gordura e os carboidratos do leite são boas fontes de energia para os idosos ${ }^{10}$. Sua gordura contém as vitaminas A e D, essenciais para o organismo humano em todas as fases da vida, inclusive na terceira idade ${ }^{8,9,10}$.

A oferta de cálcio propiciada pelo leite é necessária aos idosos tendo em vista a tendência e o risco de osteoporose $\mathrm{e}^{7,10}$. Apesar de a formação máxima de massa óssea ocorrer entre a infância e os 30 anos de idade, sua manutenção continua ao longo de toda o que torna a ingestão de cálcio essencial ${ }^{9,10}$. A vitamina D (também presente no leite) estimula a absorção do cálcio, contribuindo para manter a massa óssea adequada ${ }^{10}$.

No leite também há presença da água o que contribui para o consumo diário de líquidos dos idosos e é essencial para todos os tecidos do organismo, para estabilizar a temperatura corporal e para diversas reações metabólicas, além de facilitar o transporte de nutrientes. Muitos idosos têm dificuldades para ingerir 7 copos de água diariamente, e podem contemplar o consumo adequado de líquidos ingerindo leite todos os dias ${ }^{10}$.

Alguns estudos de revisão mostram evidências (embora não conclusivas) sobre o papel do leite na prevenção de doenças ${ }^{11,36}$. O cálcio do leite pode diminuir o risco de diabetes mellitus tipo 2 e hipertensão ${ }^{11}$, as proteínas de seu soro também podem ter efeito hipotensivo, além de possivelmente atuar na redução da gordura corporal (em conjunto com o cálcio) e do risco cardíaco $^{36}$. Como as doenças crônico-degenerativas (cardiovasculares, hipertensão, diabetes, etc.) são mais frequentes entre adultos e idosos e a alimentação é fundamental para preveni-las e tratá-las ${ }^{10}$, o leite tem se mostrado um alimen- 
to importante para reduzir a incidência destas doenças, inclusive entre os idosos ${ }^{11,36}$.

Deve-se ressaltar que o presente estudo apresenta limitações quanto à discussão da adequação do consumo de leite, tendo em vista a inexistência de recomendação para tal alimento para quaisquer faixas etárias. O Guia Alimentar para a População Brasileira não traz informações específicas para consumo desta bebida, generalizando a recomendação aos produtos lácteos. Avaliar o consumo de derivados do leite, tais como queijo e iogurte, requer atenção, pois seus derivados podem conter elevadas quantidades de gordura e açúcar ${ }^{12}$.

Porém, tendo em vista a concordância dos achados do presente trabalho com demais estudos, espera-se que os relatos obtidos re-

\section{REFERÊNCIAS}

1. IBGE - Instituto Brasileiro de Geografia e Estatística [homepage na internet]. Síntese de Indicadores Sociais, 2003. Rio de Janeiro; 2004a [atualizado em 13 abr 2004; acesso em 21 set 2009]. Disponível em:http://www.ibge.gov.br/home/ presidencia/noticias/noticia_visualiza. php?id_noticia $=132$ \&id_pagina $=1$

2. Lima-Costa MF, Veras R. Saúde pública e envelhecimento. Cad Saúde Pública. 2003; 19(3): 700-1.

3. Paz AA, Santos BRL, Eidt OR. Vulnerabilidade e envelhecimento no contexto da saúde. Acta Paul Enferm. 2006; 19(3): 338-42.

4. Almeida MHM, Gonzales MEV, Medeiros SL, Almeyda VAG, Andrade J. Relações entre envelhecimento e nutrição. Rev Bras Med. 1995; 52(6): 592-600.

5. Aranha FQ, Barros ZF, Moura LSA, Gonçalves MCR, Barros JC, Metri JC, et al. O papel da vitamina $\mathrm{C}$ sobre as alterações orgânicas no idoso. Rev Nutr. 2000; 13(2); 89-97. presentem satisfatoriamente a realidade de baixo consumo deste grupo de alimentos por parte dos entrevistados, confirmando a vulnerabilidade nutricional que acomete indivíduos idosos. A relevância deste estudo se dá pela carência de produção científica que aborde o consumo de leite na terceira idade, um assunto de grande importância para a Saúde Pública.

Considerando que a maioria dos idosos entrevistados apresentou consumo insatisfatório de leite e que isto pode acarretar conseqüências maléficas à saúde deste grupo e aos gastos dos setores de Saúde com essa população, faz-se necessário o desenvolvimento de ações voltadas para esse tema, devido às vantagens de incluir o leite na dieta diária.

6. Florentino AM. Influência dos fatores econômicos, sociais e psicológicos no estado nutricional do idoso. In: Frank AA, Soares EA. Nutrição no Envelhecer. São Paulo: Atheneu; 2002. p. 3-11.

7. Marucci MFN, Ferreira LS. Ações preventivas na terceira idade. In: Filho WJ, Gorzoni ML. Geriatria e Gerontologia: o que todos devem saber. 1. ed. São Paulo: Roca, 2008. p. 63-83.

8. Ctenas MLB. Leite longa vida: indispensável na cozinha saudável. 2. ed. São Paulo: C2 Editora e Consultoria em Nutrição; 1999.

9. Weaver CM. Should dairy be recommended as part of a healthy vegetarian diet? Point. Am J Clin Nutr. 2009; 89(Supl 5): 1634-7.

10. GENUTI - Grupo de Estudos de Nutrição na Terceira Idade. Alimentação: perguntas inquietantes \& respostas tranquilizadoras. São Paulo: Signus, 2003.

11. Martini LA, Wood RJ. Milk intake and the risk of type 2 diabetes mellitus, hypertension and prostate cancer. Arq Bras Endocrinol Metab.2009; 53(5): 688-94. 
12. Brasil. Ministério da Saúde. Secretaria de Atenção à Saúde. Coordenação-Geral da Política de Alimentação e Nutrição. Guia alimentar para a população brasileira: promovendo a alimentação saudável. Brasília; 2005

13. Philippi ST, Latterza AR, Cruz ATR, Ribeiro LC. Pirâmide alimentar adaptada: guia para escolha dos alimentos. Rev Nutr. 1999; 12(1): 1999.

14. Sachs A, Oliveira PA, Magnoni D. Riscos nutricionais na terceira idade. In: Magnoni D, Cukier C, Oliveira PA. Nutrição na terceira idade. 1. ed. São Paulo: Sarvier; 2005. p. 43-52.

15. Brasil. Ministério da Saúde. Secretaria de Vigilância em Saúde. Departamento de Vigilância Epidemiológica. Coordenação Geral do Programa Nacional de Imunizações [homepage na internet]. Campanha Nacional de Vacinação do Idoso: informe técnico. Brasília; 2006 [atualizado em 2006; acesso em 8 set 2009]. Disponível em: http://www.sbgg. org.br/profissional/artigos/pdf/ informe.pdf

16. Yamamoto LI. Caracterização do perfil nutricional de idosos participantes da Campanha de Vacinação de Idosos do Centro de Saúde Escola Geraldo de Paula Souza. In: Mostra de Alimentação e Nutrição do SUS [homepage na internet]. Anais da $2^{\text {a }}$ Mostra de Experiências BemSucedidas em Nutrição. Brasília: Coordenação-Geral da Política de Alimentação e Nutrição - CGPAN; 2008 [atualizado em 2008; acesso em 07 set 2009]. Disponível em: http:// nutricao.saude.gov.br/evento/2 mostra/ mostra_trabalho_rel.php? $\operatorname{cod}=4762$

17. Brasil. Ministério da Saúde. Estatuto do Idoso. Brasília; 2003.

18. Brito ISS, Brito CJ, Fabrini SP, Marins JCB. Caracterização das práticas de hidratação em karatecas do estado de Minas Gerais. Fit Perform J. 2006; 5(1): 24-30.

19. Cezar TM, Gianesi GCP, Rossi L. Taxa de Sudorese em Praticantes de Body Combat [periódico na internet]. 2007 [acesso em 10 set 2009]. Disponível em: $w w w$. racine.com.br/download. asp? idarquivobanco $=3041$.

20. American College of Cardiology, American Heart Association [homepage na internet]. ACC / AHA 2002 Guideline Update for Exercise Testing: a report of the American College of Cardiology/ American Heart Association. Task force on Pratical Guidelines (Committee on Exercise Testing). Washington; 2002 [atualizado em 2002; acesso em 27 ago 2009]. Disponível em: http://www. cardiosource.com/guidelines/guidelines/ exercise/exercise_clean.pdf.

21. Ryan AS, Craig LD, Finn SC. Nutrient intakes and dietary patterns of older americans: a national study. J Gerontol. 1992 47(5): M145-50.

22. Fischer JG, Johnson MA, Poon LW, Martin P. Dairy product intake of the oldest old. J Am Diet Assoc. 1995; 95(8): 918-21.

23. Elbon SM, Johnson MA, Fischer JG. Milk consumption in older Americans. Am J Public Health. 1998; 88(8): 1221-4.

24. Kurnik KF, Oliveira BJ, Oliveira MRC, Viebig RF. Estudo do consumo de leite e derivados por idosas praticantes de atividade física em uma academia no município de São Paulo. Revista Digital EFDeportes. 2010; 15(151). Disponível em: http://www.efdeportes.com/efd151/ consumo-de-leites-e-derivados-poridosas.htm

25. IBGE - Instituto Brasileiro de Geografia e Estatística [homepage na internet]. Pesquisa de Orçamentos Familiares (POF), 2002-2003. Rio de Janeiro, 2004b [atualizado em 19 maio 2004; acesso em 
23 set 2009]. Disponível em: http:// www.ibge.gov.br/home/presidencia/ noticias/noticia_impressao.php?id_noticia $=171$

26. Gerrior S, Putnam J, Bente L. Milk and milk products: their importance in the American diet. FoodReview: The Diet Quality Balancing Act. Washington; 1998. p. 29-37.

27. Lanou AJ. Should dairy be recommended as part of a healthy vegetarian diet? Counterpoint. Am J Clin Nutr. 2009; 89(Supl 5): 1638-42.

28. Beyer PL. Tratamento médico nutricional para doenças do trato gastrintestinal inferior. In: Mahan KL; Stump ES. Krause alimentos, nutrição \& dietoterapia. 12. ed. São Paulo: Roca; 2010. p. 673-706.

29. Pereira Filho D, Furlan SA. Prevalência de intolerância à lactose em função da faixa etária e do sexo: experiência do laboratório Dona Francisca, Joinville (SC). Rev Saúde e Ambiente. 2004; 5(1): 24-30.

30. Di Stefano M, Veneto G, Malservisi S, Cecchetti L, Minguzzi L, Strocchi A, et al. Lactose malabsorption and intolerance and peak bone mass. Gastroenterology. 2002; 122(7): 1793-9.

31. Sousa, DDP. Consumo de produtos lácteos informais, um perigo para a saúde pública: estudo dos fatores relacionados a esse consumo no município de Jacareí
- SP. [dissertação de mestrado]. São Paulo: Faculdade de Medicina Veterinária e Zootecnia da Universidade de São Paulo; 2005.

32. Machado FMS. Estratégias de concorrência da indústria alimentícia e seus desdobramentos na dimensão nutricional [tese de doutorado]. São Paulo: Faculdade de Saúde Pública; 2003.

33. Monteiro CA, Mondini L, Costa RBL. Mudanças na composição e adequação nutricional da dieta familiar nas áreas metropolitanas do Brasil (1988-1996). Rev Saúde Pública. 2000; 34(3): 251-8.

34. Heaney RP, McCarron DA, DawsonHughes B, Oparil S, Berga SL, Stern JS, et al. Dietary changes favorably affect bone remodeling in older adults. J Am Diet Assoc. 1999; 99(10): 1228-33.

35. Heaney RP. Calcium, dairy products and osteoporosis. J Am College Nutr. 2000; 19(Supl 2): 83-99.

36. Haraguchi FK, Abreu WC, de Paula H. Proteínas do soro do leite: composição, propriedades nutricionais, aplicações no esporte e benefícios para a saúde humana. Rev Nutr. 2006; 19(4): 479-88

Recebido em: 08/ago./2010

Modificado em 26/nov./2010 Aceito em 16/mar./2011 